\title{
Modelling and Design of State Estimator for a Pick and Place Robotic Arm
}

\author{
${ }^{1}$ Khalid K. Dandago, ${ }^{* 2}$ Ameer Mohammed, ${ }^{1}$ Osichinaka C. Ubadike, ${ }^{1}$ Mahmud S. Zango, \\ ${ }^{2}$ Abdulbasit Hassan, ${ }^{2}$ Muhammad I. Muhammad and ${ }^{2}$ Jamilu U. Yahaya \\ ${ }^{1}$ Department of Aerospace Engineering, Air Force Institute of Technology, Kaduna, Nigeria \\ ${ }^{2}$ Department of Mechatronic Engineering, Air Force Institute of Technology, Kaduna, Nigeria
} \{kkdandago | m.ameer\}@afit.edu.ng | diketronics@yahoo.com

\author{
ORIGINAL RESEARCH ARTICLE \\ Received: 23-OCT-2021; Reviewed: 04-DEC-2021; Accepted: 13-DEC-2021 \\ http://dx.doi.org/10.46792/fuoyejet.v6i4.716
}

\begin{abstract}
A robust model is essential for the design of system components such as controllers, observers state estimators, and simulators. State estimators are becoming increasingly important in modern systems, especially systems with states that may not be measured with sensors. Therefore, it is imperative to analyse the performance of different modelling and state estimator design techniques. In this research work, a parametric model of a pick and place robotic arm was obtained using system identification technique. Pick and place robotic arms have a lot of industrial applications. The parameters of the obtained model were determined using the general second-order characteristics equation and manual tuning. Furthermore, five state estimators were designed based on the developed model. The accuracy of the model, and the performance of the observers were analysed. The model was found to provide a good representation of the system. Nonetheless, with very small divergence between the model and the real system. The performance of the observers was found to be dependent on their pole locations; the higher the magnitude of the poles, the higher the state estimators' gain and the better the estimation provided. It was found out that the state estimators with high gains were more susceptible to measurement noise.
\end{abstract}

Keywords- Modelling, pick and place robots, observers, state estimators.

\section{INTRODUCTION}

$\mathrm{R}$ obotic arms have been used in industry for several years to automate strenuous, monotonous, and intricate tasks where there is a critical need for speed and precision ( $\mathrm{Li}$ et al., 2020; Ragaglia et al., 2017). Therefore, robotic arms find wide range of applications including pick and place actions, painting, assembly, part transfer, welding, coating and many more (Iqbal et al., 2012; Wei, 2020). Industrial robotic operations can lead to increase in quantity and quality of the products and decrease the amount of the discarded parts that are caused by human errors.

Pick and place robots are designed so that they can grab parts, move them to a specific direction (through translational or rotational movements), and drop them precisely in a desired position. They are usually positioned at the beginning and at the end of an assembly line cell to transfer parts to and from another assembly cell conveyer (Wei, 2020). To enhance the reliability of pick and place robots, the precision of grasping; moving and placing parts; and the safety and controllability of the robot should be improved (Berscheid et al., 2020). This will be achieved by determining the states of the robot which are quantities that defined its motion over time. The states are primarily measured using sensors.

\footnotetext{
${ }^{*}$ Corresponding Author

Section C- MECHANICAL/MECHATRONICS ENGINEERING \& RELATED SCIENCES Can be cited as:

Dandago K.K., Mohammed A., Ubadike O.C., Zango M.S., Hassan A., Muhammad M.I., and Yahaya J.U. (2021): Modelling and Design of State Estimator for a Pick and Place Robotic Arm, FUOYE Journal of Engineering and $\begin{array}{llll}\text { Technology (FUOYEJET), 6(4), 375-379. } & \end{array}$
} $\underline{\text { http://dx.doi.org/10.46792/fuoyejet.v6i4.716 }}$
However, in cases where the variables of interest cannot be measured directly or the measurements available from sensors are noisy, the states are estimated. The process of state estimation needs a good model of the system. Hence, this work evaluated the performance of different state observers and modelling techniques for a pick and place robotic manipulator.

The remaining part of the paper is organized as follows. Section 2 presents relevant works on the modelling and states measurement of a pick and place robotic arm, Sections 3 discusses the materials and methods adopted for modelling and states estimation of the robotic arm. Section 4 presents and evaluates the results obtained, with concluding remarks presented in Section 5.

\section{ReLATED WORKS}

\subsection{Modelling OF PiCK AND Place Robotic ARM}

Modelling is the process of generating the representation of a system in order to analyse its operations. In order to model a pick and place robotic arm, system identification or first principle technique-if there is complete knowledge on how the system operates-could be utilized (Gill \& D'Andrea, 2019; Hermann et al., 2017). Modelling by derivation from first principle involves the study of the forward and inverse kinematics of the robot, where the former computes the orientation and cartesian position of the arm given the kinematic configuration and the joint coordinates; On the other hand, the later involves the solution of the joint variables using the given position and orientation of robot's end effector (Iqbal et al., 2012). By utilizing the Denavit-Hartnberg parametric scheme, (Dewi et al., 2020; Iqbal et al., 2012) and (Botero-Valencia et al., 2017) all modelled a pick and place robotic manipulator using Kinematic approach.

Modelling by system identification considers the robotic arm as a black-box, whereby an input will be fed-in so as to monitor the nature of the output - the response of the 
robot. Subsequently, the relationship between the output and input will be used to derive the model of the system. For robotic arm, (Alphonse et al., 2019; Hassan et al., 2019; Rehiara, 2011) designs models using system identification. In (Hassan et al., 2019), the shaft motion and the angles of the robotic arm were the inputs of the model while the positions of the end effector (in $x, y$ and $\mathrm{z}$ coordinates) and the angles (roll, pitch and yaw) were the respective output of the model.

\subsection{Measurement of Pick and Place Robotic Arm}

The first step in controlling a robot is the determination of its states. The states of a robot can be referred to as set of quantities like orientation, velocity and position that when known or determined, describe the motion of the robot overtime. The states of most real-life systems are not readily available for use. The most basic way of determining states of a system is through use of measurement sensors. However, when the states cannot be measured directly or are susceptible to noise, they are estimated.

State estimation in real-life applications is a complex task. Over the years, the field of state estimation have experienced significant advancements including least squares, Markov chains, Kalman filter and Bayes filter development. Some of these advancements are due to ever increasing and superior computational power which enable implementation of complex computational techniques in practical systems (Barfoot, 2017). All measurements from sensors have limitations resulting from limited precision associated with the sensors. Therefore, these limitations must be accounted for in state estimation process. Generally, sensors can be categorized as interoceptive sensors (accelerometer and gyroscope) and exteroceptive sensors (camera, laser range finder, GPS transmitter/receiver). Both exteroceptive and interoceptive measurements are utilized for unsurpassed state estimation by combining measurements from multiple sensors.

Numerous works used variant procedures for state estimation and measurement for robots. The most prominent ones are the use of multi-sensor fusion and the use of Kalman filters and their derivatives, such as unscented Kalman filters, extended Kalman filters and particle filters (Al Khatib et al., 2015; Erfani et al., 2019; J.M. Li et al., 2019; Magrin \& Todt, 2019; Ruan et al., 2018; Ullah et al., 2020). In (Erfani et al., 2019) and (Al Khatib et al., 2015) IMU and GPS measurements were integrated to improve localization and navigation algorithm.

\section{Materials and Methods}

\subsection{HARDWARE PLATFORM}

A robotic arm developed by Quanser consulting company was used for the research work. The system is shown in Figure 1. The system has two main dynamics: the current-pitch angle displacement of the system and the voltage-current dynamics of its electric motor. This work focused on the current-pitch angle displacement of the robotic arm. LabVIEW software was used for obtaining the nonlinear parametric mathematical model of the pick and place robotic arm. MATLAB was utilized for state estimator design.

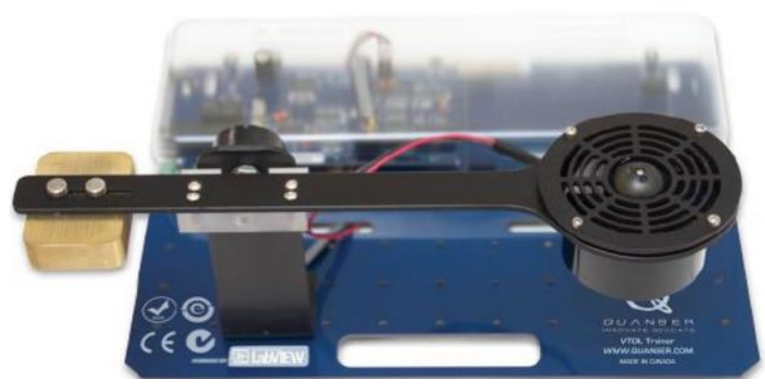

Fig. 1: A Pick and Place Robotic Arm Developed by Quanser Consulting Incorporation.

\subsection{MODELLING}

The complete model of the pick and place robotic arm was developed by model characterization and model parameters identification. These steps are discussed in the following subsections.

\subsubsection{Model Characterization}

The LabVIEW system identification toolkit was used to characterize the model of the pick and place system. The toolkit read the input given to the system - which is the motor armature current-and the corresponding response, which is the pitch angle position of the system, to characterize the model. The identified unforced model is a second order nonlinear parametric model, which was given by,

$$
\begin{gathered}
\dot{x}_{1}=f_{1}\left(x_{1}, x_{2}\right)=x_{2} \\
\dot{x}_{2}=f_{1}\left(x_{1}, x_{2}\right)=-\beta x_{2}-\gamma \sin \sin x_{2} \\
y=h\left(x_{1}, x_{2}\right)=x_{1}
\end{gathered}
$$

where $x_{1}$ is the pitch angle position, and $x_{2}$ is the angular speed of the system.

\subsubsection{Model Linearization}

For easier identification of model parameters and subsequent design of the state estimator, the nonlinear system was linearized. To obtain the equilibrium points of the unforced system, the equilibrium condition $\dot{x}_{1}=$ $\dot{x}_{2}=0$ was used. Substituting the condition into (1) and (2),

$$
x_{2}=0
$$$$
\beta(0)-\gamma \sin \sin x_{1}=0
$$

Therefore,

$$
x_{1}=(0)=0, \pi, 2 \pi, 3 \pi \ldots \ldots
$$

It is clear from (4) and (5) that equilibrium points are multiple. Therefore, the equilibrium point $\left(x_{1}, x_{2}\right)=(0,0)$ was used in linearizing the system. The linearization technique adopted was the Taylor's series expansion method. The linearized model is given by;

$$
\begin{aligned}
& \Delta \dot{x}=A \Delta x \\
& \Delta y=C \Delta x
\end{aligned}
$$

Where $\mathrm{A}$ and $\mathrm{C}$ are Jacobian matrices given by;

$$
A=\left[\begin{array}{ll}
\frac{\partial f_{1}}{\partial x_{1}} & \frac{\partial f_{1}}{\partial x_{2}} \\
\frac{\partial f_{2}}{\partial x_{1}} & \frac{\partial f_{2}}{\partial x_{2}}
\end{array}\right]_{\text {Computed with } x_{1}=x_{2}=0}
$$




$$
C=\left[\frac{\partial h}{\partial x_{1}} \frac{\partial h}{\partial x_{2}}\right]_{\text {computed with } x_{1}=x_{2}=0}
$$

Solving (8) and (9);

$$
A=\left[\begin{array}{cc}
0 & 1 \\
-\gamma & -\beta
\end{array}\right] \quad C=\left[\begin{array}{ll}
1 & 0
\end{array}\right]
$$

\subsubsection{Model Parameters Identification}

The time (T) by the pitch angle position of the system to complete one revolution was used in determining the parameter $\gamma$. This information was obtained from LabVIEW simulation. Parameter $\beta$ was then tuned until a good model of the system was obtained.

The characteristics equation of a system could be obtained from;

$$
|S I-A|=0
$$

Solving for (10), equation (11) was obtained for the system.

$$
s^{2}+\beta s+\gamma=0
$$

The general equation for second-order system is given by (12).

$$
s^{2}+2 \zeta \omega_{n} s+\omega_{n}^{2}=0
$$

Comparing (11) with (12); $\gamma=\omega_{n}{ }^{2}$

Where $\quad \omega_{n}=\left(\frac{2 \pi}{T}\right)^{2}$ and $T=1.7$; Therefore, $\quad \gamma=13.66$. Parameter $\beta$ was tuned until a model that fits the system was obtained. The value that did that was $\beta=0.8$.

\subsection{Observer (State Estimator) Design}

The first step in designing a state estimator, (or observer), is to find out whether the system is observable or not. The observability matrix $\left(\mathrm{O}_{\mathrm{m}}\right)$ is given by;

$$
\begin{aligned}
& \left.O_{m}=\left[\begin{array}{c}
C \\
C A
\end{array}\right]=\left[\begin{array}{cc}
{\left[\begin{array}{cc}
1 & 0
\end{array}\right]} & \\
{[1} & 0
\end{array}\right]\left[\begin{array}{cc}
0 & 1 \\
-13.66 & -0.8
\end{array}\right]\right]=\left[\begin{array}{ll}
1 & 0 \\
0 & 1
\end{array}\right]\left|O_{m}\right| \\
& =1
\end{aligned}
$$

The system is observable since $\left|O_{m}\right| \neq 0$. Hence, it is possible to estimate the states of the system with designed estimators. Acker, which is a MATLAB command, was used in designing the state estimators. The main MATLAB statement that did that was;

$$
L=\operatorname{acker}\left(A^{\prime}, C^{\prime}, P\right)^{\prime}
$$

Where $\mathrm{L}$ is the observer gains and $\mathrm{P}$ is the poles locations of the observer.

\section{RESULTS AND DISCUSSIONS}

This section presents and discusses the results obtained from the model and state estimator.

\subsection{MODELLING RESULTS}

The identified parameters of the parametric model were obtained as: $\gamma=13.66$ and $\beta=0.8$. The response of the real system, which was represented as measured, was compared with that of the model-which was represented as simulated - as shown in Figure 2.

In Figure 2, the unforced system was perturbed; it can be observed that the model provides a good representation of the system. However, there is an apparent discrepancy, of less than $5 \%$, between the responses. One of the reasons for the discrepancy is the approximation made using the Taylor's series linearization method. That is, higher order derivative terms were neglected because they were relatively smaller than the lower order terms. Another reason could be the manual tuning approach adopted for identifying $\beta$. Furthermore, possible internal and external disturbances, such as friction, that could affect the real system might lead to the discrepancy. Moreover, sensor error can contribute in the mismatch.

\subsection{State Estimator Results}

Four estimators were designed to estimate the pitch $\left(x_{1}\right)$ and speed $\left(x_{2}\right)$ of the pick and place robotic arm. The estimators' gains with their respective pole locations are given by Table 1 . It could be seen from Figure 3 that the higher the magnitude of the poles the better the estimation of the pitch by the state estimators.

In Figure 3, the red plots represent the sensor measurement of the of the pitch position, while the blue plots are the pitch estimates by the observers. The pink plots depict the speed of the system.

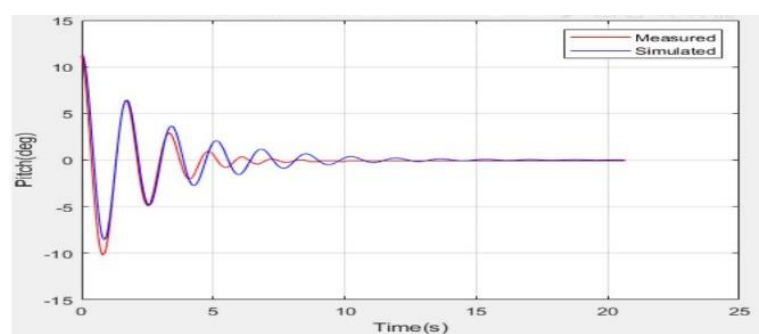

Fig. 2: Comparison of the Real System Response with Model Response

Although poles with larger magnitude in the negative plane estimates the states better, they have larger bandwidth which make them susceptible to sensor noise. All measurements used for state estimation come with noise, and it could be observed from Table 1 that higher the magnitude of the of the poles the higher the observer gains. Therefore, poles that are farther away in the negative axis will amplify the noise higher. This problem could be seen from the transient response in Figure 4. Figure 4 presents the estimation made by an observer with gains at [-100+100j, $-100-100 j]$. Therefore, appropriate considerations should be made to have a good balance between state estimator error elimination and measurement noise reduction.

Table1. Estimator Gains and their Pole Locations.

\begin{tabular}{ccc}
\hline $\begin{array}{c}\text { State } \\
\text { Estimators }\end{array}$ & $\begin{array}{c}\text { State Estimators' } \\
\text { Gains }\end{array}$ & Pole Locations \\
\hline Observer 1 & {$[2.2-13.42]$} & {$[-1-2]$} \\
Observer 2 & {$[1.2-12.62]$} & {$[-1+j-1-j]$} \\
Observer 3 & {$[19.2170 .98]$} & {$[-10+10 j-10$} \\
& & $-10 j]$ \\
Observer 4 & {$[39.2754 .98]$} & {$[-20+20 j 20$} \\
\hline
\end{tabular}



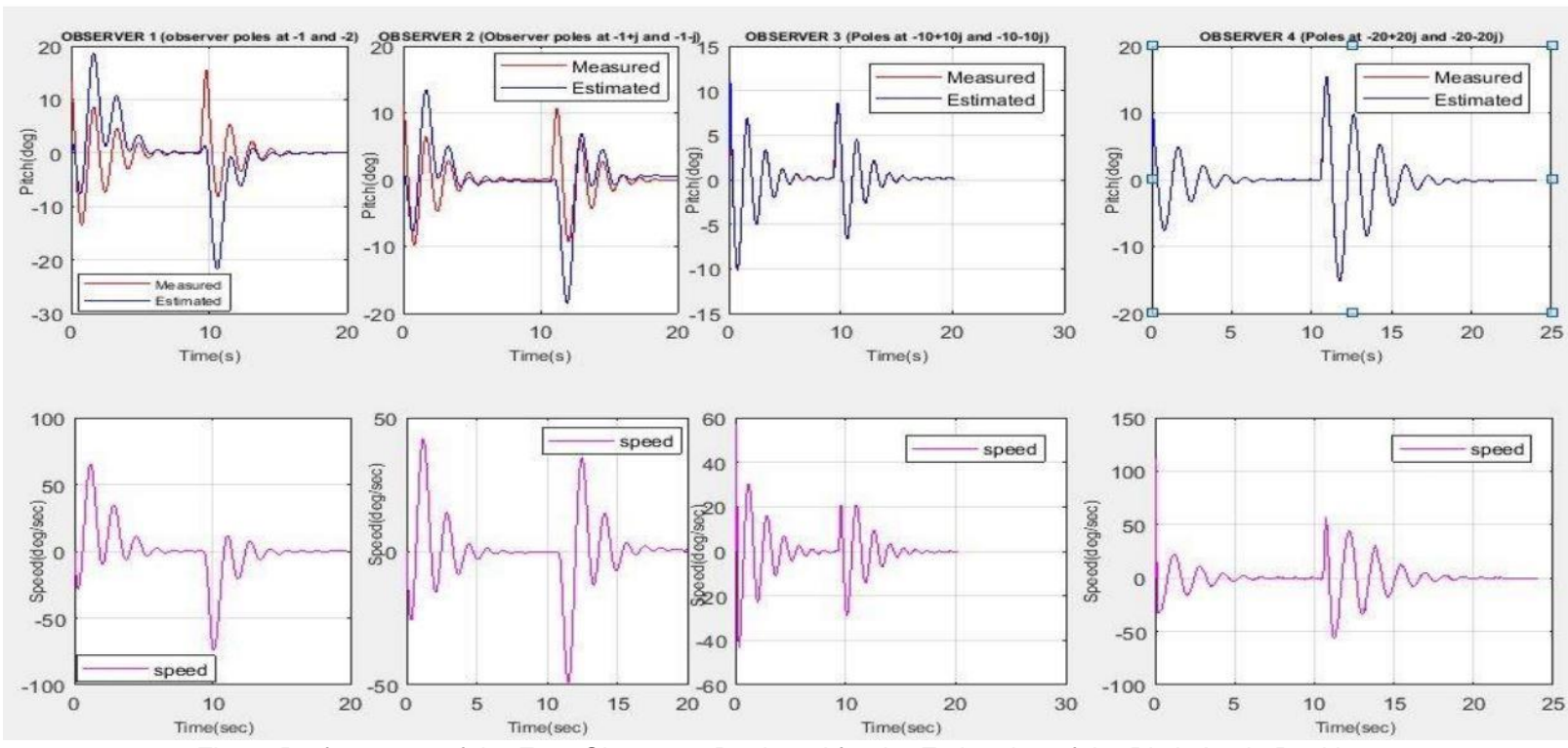

Fig. 3: Performance of the Four Observers Designed for the Estimation of the Pitch Angle Position.
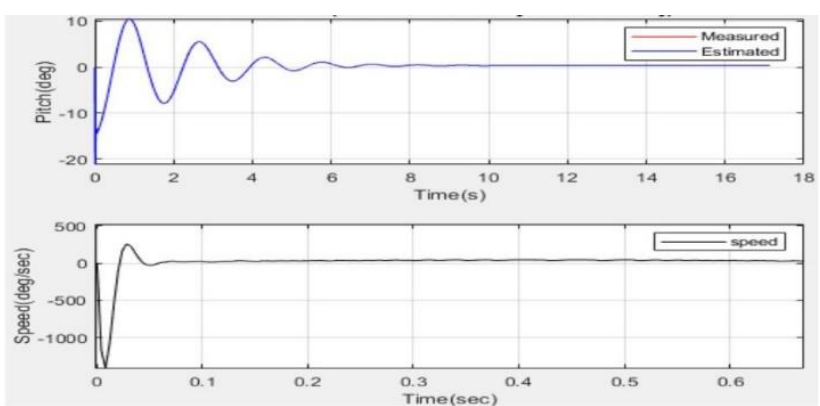

Fig. 4: Performance of an Observer that has its Poles at $[-100+100]$, $-100-100 j]$.

\section{ConcLusion}

The modelling and state estimator design of a pick and place robotic arm was presented. Both system identification and first principle techniques were incorporated to obtain a model that gave a good depiction of the robotic arm. The inevitable mismatch between the model and the real system, which was found to be less than $5 \%$, was discussed. Furthermore, observers that gave a good estimation of the system's states were developed. It was observed that there must be proper trade-off between accuracy and noise elimination in the state estimation design.

Future works should consider the analysis of nonparametric model of the same system to assess the model that would give better performance. Also, robust filters should be added to the state estimator design part for better noise elimination in the estimations.

\section{REFERENCES}

Al Khatib, E., Jaradat, M., Abdel-Hafez, M., \& Roigari, M. (2015). Multiple sensor fusion for mobile robot localization and navigation using the Extended Kalman Filter. 10th International Symposium on Mechatronics and its Applications (ISMA), 1-5, https://doi.org/10.1109/ISMA.2015.7373480

Alphonse, A. A., Abbas, A. A., Fathy, A. M., Elsayed, N. S., Ammar, H. H., \& Elsamanty, M. M. (2019). Modelling of continuum robotic arm using artificial neural network (ANN). 2019 Novel Intelligent and Leading Emerging Sciences Conference (NILES), 1, 191-195.

Barfoot, T. D. (2017). State estimation for robotics. Cambridge
University Press. https://doi.org/10.1017/9781316671528

Berscheid, L., Meißner, P., \& Kröger, T. (2020). Self-supervised Learning for Precise Pick-and-place without Object Model. IEEE/RSJ International Conference on Intelligent Robots and Systems.

October 25-29, 2020, Las Vegas, NV, USA

Botero-Valencia, J., Marquez-Viloria, D., Castano-Londono, L., \& Morantes-Guzmán, L. (2017). A low-cost platform based on a robotic arm for parameters estimation of Inertial Measurement Units. Measurement, 110, 257-262. https://doi.org/10.1016/j.measurement.2017.07.002

Dewi, T., Nurmaini, S., Risma, P., Oktarina, Y., \& Roriz, M. (2020). Inverse kinematic analysis of 4 DOF pick and place arm robot manipulator using fuzzy logic controller. International Journal of Electrical and Computer Engineering (IJECE), 10, 1376-1386. https://doi.org/10.11591/ijece.v10i2.pp1376-1386

Erfani, S., Jafari, A., \& Hajiahmad, A. (2019). Comparison of two data fusion methods for localization of wheeled mobile robot in farm conditions. Artificial Intelligence in Agriculture, 1, 48-55. https://doi.org/https://doi.org/10.1016/j.aiia.2019.05.002

Gill, R., \& D'Andrea, R. (2019). Computationally Efficient Force and Moment Models for Propellers in UAV Forward Flight Applications. In Drones (Vol. 3, Issue 4). https://doi.org/10.3390/drones3040077

Hassan, H., Abbas, A., \& Demian, A. (2019). Modelling of Continuum Robotic Arm Using Artificial Neural Network (ANN). https://doi.org/10.1109/NILES.2019.8909308

Hermann, J., Distasio, R., \& Tkatchenko, A. (2017). First-Principles Models for van der Waals Interactions in Molecules and Materials: Concepts, Theory, and Applications. Chemical Reviews, 117. https://doi.org/10.1021/acs.chemrev.6b00446

Iqbal, J., Ul Islam, M., \& Khan, H. (2012). Modelling and analysis of a 6 DOF robotic arm manipulator. Canadian Journal on Electrical and Electronics Engineering, 3, 300-306.

Li, F., Jiang, Y., Li, T., Feng, Y., \& Chen, S. (2020). Design of a robot end effector with measurement system for precise pick-andplace of square objects. Procedia Manufacturing, 48, 172-180. https://doi.org/10.1016/j.promfg.2020.05.035

Li, J.-M., Chen, C., \& Cheng, T.-H. (2019). Estimation and Tracking of a Moving Target by Unmanned Aerial Vehicles. 2019 American Control Conference (ACC), 2019, 3944-3949. https://doi.org/10.23919/ACC.2019.8815101

Magrin, C., \& Todt, E. (2019). Multi-Sensor Fusion Method Based on Artificial Neural Network for Mobile Robot Self-Localization. 2019 Latin American Robotics Symposium (LARS), 2019 Brazilian Symposium on Robotics (SBR) and 2019 Workshop on Robotics in Education (WRE), 138-143. https://doi.org/10.1109/LARS-SBR- 
WRE48964.2019.00032

Ragaglia, M., Maria, A., \& Rocco, P. (2017). Trajectory generation algorithm for safe human-robot collaboration based on multiple depth sensor measurements. Mechatronics, 55, 267-281. https://doi.org/10.1016/j.mechatronics.2017.12.009

Rehiara, A. (2011). System Identification Solution for Developing an Adept Three Robot Arm Model. Cyber Journals, 1-5.

Ruan, X., Liu, S., Ren, D., \& Zhu, X. (2018). Accurate 2D Localization for Mobile Robot by Multi-sensor Fusion. 2018 IEEE 4th Information Technology and Mechatronics Engineering Conference (ITOEC), 839-843. https://doi.org/10.1109/ITOEC.2018.8740490

Ullah, I., Su, X., Zhang, X., \& Choi, D. (2020). Simultaneous Localization and Mapping Based on Kalman Filter and Extended Kalman Filter. Wireless Communications and Mobile Computing, 2020, 2138643. https://doi.org/10.1155/2020/2138643

Wei, B. (2020). Design and Modelling of a Pick and Place Robotic Manipulator, Proceedings of the 7th International Conference of Control Systems, and Robotics (CDSR'20) Virtual Conference, November, 2020. https://doi.org/10.11159/cdsr20.154 